\title{
Resource Allocation in Full-Duplex UAV Enabled Multi-Small Cell Networks
}

\author{
Amirhosein Hajihoseini Gazestani, Seyed Ali Ghorashi, Senior Member, IEEE, Zhaohui Yang, \\ and Mohammad Shikh-Bahaei, Senior Member, IEEE
}

\begin{abstract}
Flying platforms such as Unmanned Aerial Vehicles (UAVs) are a promising solution for future small cell networks. UAVs can be used as aerial Base Stations (BSs) to enhance coverage, capacity and reliability of wireless networks. Also, with recent advances of Self Interference Cancellation (SIC) techniques in Full-Duplex (FD) systems, practical implementation of FD BSs is feasible. In this paper, we investigate the problem of resource allocation for multi-small cell networks with FD-UAVs as aerial BSs with imperfect SIC. We consider three different scenarios: a) maximizing the DL sum-rate, b) maximizing the UL sum-rate, and finally c) maximizing the sum of UL and DL sum-rates. The aforementioned problems result in non-convex optimization problems, therefore, successive convex approximation algorithms are developed by leveraging D.C. (Difference of Convex functions) programming to find sub-optimal solutions. Simulation results illustrated validity and effectiveness of the proposed radio resource management algorithms in comparison with ground BSs, in both FD mode and its half-duplex (HD) counterpart. The results also indicate those situations where using aerial BS is advantageous over ground BS and reveal how FD transmission enhances the network performance in comparison with HD one.
\end{abstract}

Index Terms-Full-Duplex (FD), Unmanned Aerial Vehicle (UAV), Resource Allocation, D.C. Programming, Power Management.

\section{INTRODUCTION}

$\mathrm{R}$ ECENTLY Unmanned Aerial Vehicle (UAV) communications have attracted researchers' attention [1], [2], [3]. When events like earthquake occur and the communication infrastructures are destroyed, UAVs can play an important role as a temporary aerial Base Station (BS) to achieve rapid service recovery. Besides, UAVs can dynamically reposition themselves to improve coverage, spectral efficiency and user quality of experience in comparison with ground BSs which have fixed locations [4], [5]. Compared to the ground BSs, UAVs can adjust their altitude, avoid obstacles and enhance the likelihood of establishing the line-of-sight connection to the ground users [3], [6], [7], |8|. Therefore, UAV communications have been considered as a promising technology for the fifth-generation (5G) of cellular network technology and beyond [1], [9], [10]. Due to the spectrum scarcity, inband Full-Duplex (FD) communication has attracted attention, because it increases the throughput and capacity in comparison with Half-Duplex (HD) counterpart, by sending and receiving data in the same frequency band and the same time [11], [12], [13]. To achieve advantages of $\mathrm{FD}$

- A. Hajihoseini Gazestani is with the Cognitive Telecommunication Research Group, Department of Electrical Engineering, Shahid Beheshti University G. C., Tehran, Iran.

- S. A. Ghorashi is with the School of Architecture, Computing and Engineering, University of East London, London, UK. He is also with the Cognitive Telecommunication Research Group, Department of Electrical Engineering, Shahid Beheshti University G. C., Tehran, Iran. E-mail:s.a.ghorashi@uel.ac.uk

- Z. Yang and M. Shikh-Bahaei are with the Centre for Telecommunications Research, King's College London, London, UK.

- This work was partly supported by the Engineering and Physical Science Research Council (EPSRC) through the Scalable Full-Duplex Dense Wireless Networks (SENSE) grant EP/P003486/1.

Manuscript received ? ?, 2019; revised ? ?, ?. communication, the most important challenge is how to cancel the self-interference (SI) [14]. Recent advances in SI Cancellation (SIC) techniques achieves more than $110 d B$ SI reduction [15], [16], [17], therefore, FD communication can be considered for the next generations of wireless networks. As a result, several works have investigated the achievable performance of FD in various applications which are presented in detail in [18].

One of the important applications of FD technology is to increase the capacity of the network for cellular BSs. One small cell with FD BS and multiple HD users are considered in [11]. The authors formulated a joint power allocation and user pairing problem to maximize the total sum-rate. The problem is divided into two subproblems using a novel decomposition method. Finally, results are compared with the greedy algorithm and random pairing method. In real scenarios, usually, there are more than one single cell and hence, the intra-cell interference is added to the SI and inter-cell interference. Unlike [11] which has not considered multi-cell and intra-cell interference, the authors in [19] considered the FD system in small multi-cell networks where BSs are armed with FD technology and users operate in HD mode. The authors maximized the sum of uplink (UL) and downlink (DL) sum-rates considering SI, inter-cell and intra-cell interference. A joint resource and power allocation algorithm is presented to assign resource blocks for FD operation and pair users with appropriate power levels. The authors compared the results with HD mode and mentioned that the performance limiting factor is intra-cell interference. The mentioned papers consider ground BSs which support limited coverage area, while using UAVs as aerial BSs can be a promising solution for future networks to increase the capacity and coverage of small cells [4], |6].

The problem of resource allocation for UAV enabled 
vehicular communication was considered in [20] where UAVs work as cellular BSs to serve cellular and vehicle-tovehicle communication users. The authors maximized the sum achievable rate of vehicle to infrastructure communications and ensured the reliability of vehicle-to-vehicle communications. In addition, the control of the UAV trajectory is obtained using Q-Learning to adapt to the time-varying channel. An UL power control for UAV assisted network was investigated in [21]. The authors considered a UAV which serves UL users. The altitude, antenna beamwidth, UAV's location, bandwidth and power of users are jointly optimized to minimize the sum UL power, while considering the minimal rate demand. Unlike [20], [21] which considers UAVs in a single-cell scenario, authors in [22] considered UAVs as aerial BSs as a solution for deploying dense networks. The authors assumed that UAVs and ground BSs coexist and then maximized user satisfaction with provided data rates by finding the optimal position of UAVs and associating users to ground BS or UAVs. Authors in [20], [21], [22] employed HD transmission in UAVs, while authors in [23] investigated the problem of 3-D drone BS placement with FD communication in the heterogeneous networks. The authors employed FD drones in coexistence with ground BS to improve network throughput. The authors assumed different frequency spectra for drone BSs and hence, there is no interference between drones. We review networks with ground FD BSs, aerial HD BSs and aerial FD BSs without frequency reuse and intra-cell interference. This literature review is summarized in Table 1 .

In this paper, we consider multiple FD small cell networks in which each cell is covered by a UAV as an aerial BS. We assume that UAVs are armed with FD technology (FDUAV) with imperfect SIC to serve both UL and DL users at the same time and the same frequency band, simultaneously while users operate in HD mode because of hardware limitation. Since in this setting, FD technology is used in multismall cell networks, DL and UL transmission coexist, and we have to consider SI and inter-cell interference. Moreover, unlike [23] which considers different frequency spectrum for each BS, we consider frequency reuse in cells in order to increase the network capacity and data rate, hence, we have to consider intra-cell interference. Here, we investigate three different scenarios; at first, we aim to maximize DL sum-rate whilst prescribing a certain minimum requirement for the UL transmission rate by optimizing FD-UAV transmission power. In the second scenario, we aim to maximize UL sum-rate whilst prescribing a certain minimum requirement for the DL transmission rate by optimizing the transmission power of users. Finally, we aim to maximize the sum of DL and UL sum-rates by jointly optimizing the transmission power of FD-UAV and users. These optimization problems are non-convex, therefore, a successive convex approximation algorithm is developed by leveraging the D.C. (Difference of Convex functions) programming to find sub-optimal solutions. In each scenario, we compare the performance of FD-UAV with ground BS, in both FD and HD modes, to show the effectiveness of using FD-UAVs as aerial BSs. The main contributions of this paper are as follows:

- Employing FD-UAV with imperfect SIC as an aerial BS in multi-small cell networks, assuming frequency reuse and intra-cell interference.

- Analyzing network sum-rate in three different objective functions.

- Finding suboptimal solutions for resource allocation problem by leveraging D.C. programming, considering SI, inter-cell and intra-cell interferences.

- Evaluating and comparing the performance of aerial BSs with ground BSs, in both FD and HD modes.

The rest of the paper is organized as follows: In Section 2 we describe the system model and problem formulation. In Section 3 we investigate three different scenarios, DL sum-rate analysis, UL sum-rate analysis and sum of UL and DL sum-rate analysis. Simulation results are presented and discussed in Section 4 to evaluate the performance of the proposed system model and radio resource management method and finally, the paper is concluded in Section 5 .

The parameters commonly used throughout the paper are presented in Table 2

\section{System Model and Problem Formulation}

Consider a cellular network with a central unit and several small cells which are covered by UAVs as aerial BSs. It is assumed that UAVs are armed with FD capability to send and receive data in the same frequency band, simultaneously, while users operate in traditional HD transmission. Each FD-UAV has $T$ frequency channels and can support $T$ UL and $T$ DL users, simultaneously. The main problem in FD systems is SIC. To model this, we assume that SIC is performed on FD-UAV imperfectly with residual SI to power ratio of $\beta\left[19\right.$, p. 2]. This means that if the $m^{t h}$ FD-UAV transmits in the $n^{\text {th }}$ channel with the power of $P_{m n}^{U A V}$, the residual SI is $\beta P_{m n}^{U A V}$ and parameter $\beta$ quantifies the amount of SIC; when $\beta=0$, there is perfect SIC while for $\beta=1$ there is no SIC. The $m^{\text {th }}$ FD-UAV is located at $y_{m}=\left(y_{m}(1), y_{m}(2), H_{m}\right), m=1,2, \ldots, M$ in three-dimensional space where $M$ is the total number of cells. In addition, in order to consider the worst-case interference scenario, we assume that there are $N=M T$ UL and $N=M T$ DL users where the $n^{t h} \mathrm{UL}$ and $n^{\text {th }}$ DL users are located at $x_{n}=\left(x_{n}(1), x_{n}(2), 0\right)$ and $z_{n}=\left(z_{n}(1), z_{n}(2), 0\right), n=1,2, \ldots, N$, respectively. This system model is represented in Fig. 1] Moreover, we assume that users are located outdoors in rural areas and channel links are assumed to be block fading and remain unchanged in the scheduling process. The communication channel between FD-UAV and each user is dominated by a LOS path, hence, the channel between the $m^{t h}$ FD-UAV and the $n^{t h}$ UL user and the channel between the $n^{\text {th }}$ UL and DL users are $\frac{h_{0}}{D_{y m-x_{n}}^{\alpha_{1}}}$ and $\frac{h_{0} g}{D_{z_{n}}^{\alpha_{2}-x_{n}}}$ respectively, where $h_{0}$ is the power gain at the reference distance $1 m, g$ is the small scale fading coefficient and $D_{y_{m}-x_{n}}=\left\|y_{m}-x_{n}\right\|$. Moreover, $\alpha_{1}$ and $\alpha_{2}$ are the path-loss exponents for air to ground and ground to ground communication, respectively.

Without loss of generality, we assume that the FD-UAV is equipped with a directional antenna with adjustable beamwidth and each user is equipped with an omnidirectional antenna with unit gain. The half-power beamwidth of the FD-UAV is denoted by $2 \Theta \in(0, \pi)$, therefore, the 
TABLE 1: Summary of Scenarios of the Literature Review

\begin{tabular}{|c|c|c|c|c|c|}
\hline Reference \# & BS Type & $\begin{array}{l}\text { Transmission } \\
\text { Mode }\end{array}$ & Cell Type & Objective Function & Optimization Variable \\
\hline 11 & Ground & FD & Single & Total sum-rate & User pairing and power allocation \\
\hline$\overline{19}$ & Ground & FD & Multiple & Total sum-rate & Radio resource management \\
\hline 20 & Aerial & HD & Single & Sum achievable rate & Resource allocation and UAV trajectory control \\
\hline |21 | & Aerial & HD & Single & UL sum power & $\begin{array}{l}\text { Altitude, beamwidth, location and bandwidth } \\
\text { optimization }\end{array}$ \\
\hline |22| & $\begin{array}{l}\text { Ground } \\
\& \text { Aerial }\end{array}$ & HD & Multiple & $\begin{array}{l}\text { User satisfaction with provided } \\
\text { data rate }\end{array}$ & UAV positioning and user association \\
\hline |23| & Aerial & FD & Multiple & Network throughput & $\begin{array}{l}\text { UAV placement and resource allocation without } \\
\text { frequency reuse assumption }\end{array}$ \\
\hline Proposed & Aerial & FD & Multiple & $\begin{array}{l}\text { DL sum-rate, UL sum-rate and total } \\
\text { sum-rate }\end{array}$ & Resource allocation assuming frequency reuse \\
\hline
\end{tabular}

antenna gains for the $m^{\text {th }} \mathrm{UAV}$ which is connected to the $n^{\text {th }}$ user (or the $n^{\text {th }} \mathrm{UAV}$ ) can be modeled as [24]:

$$
G_{m n}^{t}=\left\{\begin{array}{cc}
\frac{G_{0}}{\Theta^{2}} & \text { if } 0 \leqslant \theta_{m n} \leqslant \Theta \\
g_{0} \approx 0 & \text { otherwise, }
\end{array}\right.
$$

where $t \in\{U L, D L, D\}$ indicates communication between FD-UAV and UL user, FD-UAV and DL user and FD-UAV with another FD-UAV, respectively, and $\theta_{m n}$ is the azimuth angle between the $m^{t h}$ FD-UAV and $n^{t h}$ user (or $n^{\text {th }}$ FD$\mathrm{UAV})$. In addition, $G_{0} \approx 2.2846$ and $g_{0}$ means the channel

\section{TABLE 2: Frequent Parameters}

\begin{tabular}{|c|c|}
\hline Parameter & Description \\
\hline$N$ & Number of DL/UL users \\
\hline $\bar{M}$ & Number of FD-UAVs (small cells) \\
\hline$T$ & Number of frequency channels \\
\hline$H_{m}$ & Height of the $m^{t h}$ FD-UAV \\
\hline$y_{m}$ & $\begin{array}{l}\text { Location of the } m^{t h} \text { FD-UAV in three-dimensional } \\
\text { space }\end{array}$ \\
\hline$x_{n}$ & $\begin{array}{l}\text { Location of the } n^{t h} \text { UL user in three-dimensional } \\
\text { space }\end{array}$ \\
\hline$z_{n}$ & $\begin{array}{l}\text { Location of the } n^{t h} \text { DL user in three-dimensional } \\
\text { space }\end{array}$ \\
\hline$\beta$ & The ratio of residual SI to power \\
\hline$P_{m n}^{U A V}$ & $\begin{array}{l}\text { Transmitting power of the } m^{t h} \text { FD-UAV in the } n^{t h} \\
\text { channel }\end{array}$ \\
\hline$P_{\max }^{U A V}$ & Maximum power of FD-UAV \\
\hline$P_{m n}^{U E}$ & $\begin{array}{l}\text { Transmitting power of the } n^{t h} \text { UL user which is } \\
\text { connected to the } m^{t h} \text { FD-UAV }\end{array}$ \\
\hline$P_{\max }^{U E}$ & Maximum power of UL users \\
\hline$h_{0}$ & Power gain at the reference distance $1 m$ \\
\hline$g$ & Fading coefficient \\
\hline$D_{y_{m}-x_{n}}$ & Distance between $y_{m}$ and $x_{n}$ \\
\hline$\alpha_{1}$ & $\begin{array}{l}\text { Path-loss exponent for air to ground channel and vice } \\
\text { versa }\end{array}$ \\
\hline$\alpha_{2}$ & Path-loss exponent for the ground to ground channel \\
\hline $2 \Theta$ & Half-power beamwidth \\
\hline$\theta_{m n}$ & $\begin{array}{l}\text { Azimuth angle between the } m^{t h} \text { FD-UAV and the } \\
n^{t h} \text { user }\end{array}$ \\
\hline$G_{m n}$ & $\begin{array}{l}\text { Antenna gain for the } m^{t h} \text { FD-UAV which is con- } \\
\text { nected to the } n^{t h} \text { user }\end{array}$ \\
\hline$G_{m m^{\prime}}$ & $\begin{array}{l}\text { Antenna gain for the } m^{t h} \text { FD-UAV which is con- } \\
\text { nected to the } m^{t h} \text { FD-UAV }\end{array}$ \\
\hline$g_{0}$ & Channel gain outside of the antenna beamwidth \\
\hline$b_{m n}$ & $\begin{array}{l}\text { Indicates the connectivity between the } m^{t h} \text { FD-UAV } \\
\text { and the } n^{t h} \text { user }\end{array}$ \\
\hline$w$ & The bandwidth of each frequency channel \\
\hline$\overline{\sigma_{N}^{2}}$ & Noise power \\
\hline$\sigma_{I}^{2}$ & Intra-cell interference power \\
\hline$R_{\min }^{U L}$ & Minimum of UL rate demand \\
\hline$R_{\min }^{D L}$ & Minimum of DL rate demand \\
\hline
\end{tabular}

gain outside the beamwidth of the antenna. We should notice that the parameter $G_{m n}^{t}$ is a function of user and FDUAV locations, if users are in the coverage area of FD-UAV, $G_{m n}^{t}$ is nonzero, while if users are not in the coverage area, $G_{m n}^{t}$ is zero and FD-UAV cannot communicate with the user. Besides, if two FD-UAVs want to communicate with each other, they should be in the coverage area of each other. For example, in Fig. 1 the $2^{\text {nd }}$ FD-UAV does not receive any interference signal from two other FD-UAVs. Moreover, the DL user which is connected to the $2^{\text {nd }}$ FD-UAV does not receive any interference from the $3^{\text {rd }}$ FD-UAV while receives interference from the $1^{\text {st }}$ FD-UAV.

\subsection{FD-UAV Altitude}

In order to determine the altitude of the $m^{t h}$ FD-UAV, we consider a predetermined value for half-power beamwidth of FD-UAV, i.e., $\Theta=\Theta_{0}$, then calculate the altitude of the $m^{t h}$ FD-UAV. The altitude of the $m^{t h}$ FD-UAV can be calculated as:

$$
\begin{aligned}
& H_{m 0}=\max \left\{\frac{\max \left(b_{m n} D_{y_{m}-x_{n}}\right)}{\tan \Theta_{0}}, \frac{\max \left(b_{m n} D_{y_{m}-z_{n}}\right)}{\tan \Theta_{0}}\right\} \\
& H_{L} \leqslant H_{m 0} \leqslant H_{U} \text {, }
\end{aligned}
$$

where $b_{m n} \in\{0,1\}$ indicates that the $n^{\text {th }}$ user is connected to the $m^{\text {th }}$ FD-UAV or not. By considering practical aspects, the FD-UAV altitude should be bounded between $H_{L}$ and $H_{U}$. If $H_{m 0}$ becomes lower than its lower bound, we set its value equal to its lower bound, and if it becomes more than its upper bound, we set its value equal to its upper bound.

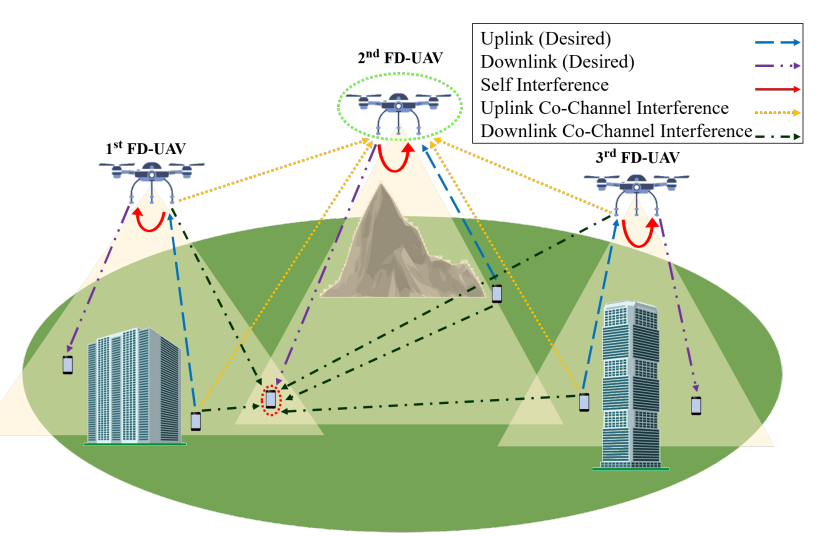

Fig. 1: UL, DL and interference signals in the system model including UL and DL users with three FD-UAVs. 


\subsection{Problem Formulation}

We assume that FD-UAVs work in the same frequency bands using frequency reuse. In addition, we assume that each FD-UAV has $T$ frequency channels and the channel allocation is predetermined. In each channel, one DL user and one UL user communicate with FD-UAV, simultaneously. We divide the users into $M$ groups and for each group, we put a FD-UAV as aerial BS. The received signal to interference plus noise ratio (SINR) at the $m^{\text {th }}$ FD-UAV for the $n^{\text {th }}$ user and SINR at the $n^{\text {th }}$ DL user which is connected to the $m^{t h}$ FD-UAV can be expressed as (3) and (4), respectively.

$$
\begin{gathered}
\Gamma_{m n}^{U L}=\frac{P_{m n}^{U E} D_{y_{m}-x_{n}}^{-\alpha_{1}} h_{0} G_{m n}^{U L}}{\sigma_{N}^{2}+\beta P_{m n}^{U A V}+I_{U A V}^{U L}+I_{U E}^{U L}}, \\
\Gamma_{m n}^{D L}=\frac{P_{m n}^{U A V} D_{y_{m}-z_{n}}^{-\alpha_{1}} h_{0} G_{m n}^{D L}}{\sigma_{N}^{2}+I_{U A V}^{D L}+I_{U E}^{D L}},
\end{gathered}
$$

where

$$
\begin{aligned}
I_{U A V}^{U L} & =\sum_{m^{\prime}=1, m^{\prime} \neq m}^{M} P_{m^{\prime} n}^{U A V} D_{y_{m}-y_{m^{\prime}}}^{-\alpha_{1}} h_{0}\left(G_{m m^{\prime}}^{D}\right)^{2}, \\
I_{U E}^{U L} & =\sum_{m^{\prime}=1, m^{\prime} \neq m}^{M} P_{m^{\prime} n}^{U E} D_{y_{m^{\prime}}-x_{n}}^{-\alpha_{1}} h_{0} G_{m^{\prime} n}^{U L}, \\
I_{U A V}^{D L} & =\sum_{m^{\prime}=1, m^{\prime} \neq m}^{M} P_{m^{\prime} n}^{U A V} D_{y_{m^{\prime}}-z_{n}}^{-\alpha_{1}} h_{0} G_{m^{\prime} n}^{D L}, \\
I_{U E}^{D L} & =\sum_{m^{\prime}=1}^{M} P_{m^{\prime} n}^{U E} D_{x_{n}-z_{n}}^{-\alpha_{2}} h_{0} g .
\end{aligned}
$$

$P_{m n}^{U E}$ is transmitting power of the $n^{t h}$ UL user which is connected to the $m^{t h}$ FD-UAV, $P_{m n}^{U A V}$ is transmitting power of the $m^{t h}$ FD-UAV for the $n^{t h}$ user, $\beta$ models SIC at FD-UAV and $\sigma_{N}^{2}$ is the noise power. In addition, we should mention that the channel between UAV and ground users depends on channel power gain at reference distance $\left(h_{0}\right)$, fading coefficient $(g)$ antenna gain and halfpower beamwidth $\left(G_{m n}^{t}\right.$ and $\left.\Theta\right)$, path-loss exponent $\left(\alpha_{1}\right)$, location of users $\left(x_{n}\right.$ or $\left.z_{n}\right)$, and finally the location and altitude of $\operatorname{UAV}\left(D_{y_{m}-x_{n}}\right)$ where $D_{y_{m}-x_{n}}=\left\|\underline{y}_{m}-\underline{x_{n}}\right\|=$ $\sqrt{\left(y_{m}(1)-x_{n}(1)\right)^{2}+\left(y_{m}(2)-x_{n}(2)\right)^{2}+H_{m}{ }^{2}}$. Without loss of generality and for the sake of simplicity, we model interference signals from other cells by $\sigma_{I}^{2}$ which determines interference power. Hence, (3) and (4) can be rewritten as (5) and (6), respectively.

$$
\begin{gathered}
\Gamma_{m n}^{U L}=\frac{P_{m n}^{U E} D_{y_{m}-x_{n}}^{-\alpha_{1}} h_{0} G_{m n}^{U L}}{\sigma_{N}^{2}+\beta P_{m n}^{U A V}+\sigma_{I}^{2}}, \\
\Gamma_{m n}^{D L}=\frac{P_{m n}^{U A V} D_{y_{m}-z_{n}}^{-\alpha_{1}} h_{0} G_{m n}^{D L}}{\sigma_{N}^{2}+P_{m n}^{U E} D_{x_{n}-z_{n}}^{-\alpha_{2}} h_{0} g+\sigma_{I}^{2}} .
\end{gathered}
$$

Now, the UL and DL rates are given, respectively, by (7) and (8):

$$
\begin{aligned}
& R_{m n}^{U L}=b_{m n} w \log _{2}\left(1+\Gamma_{m n}^{U L}\right), \\
& R_{m n}^{D L}=b_{m n} w \log _{2}\left(1+\Gamma_{m n}^{D L}\right),
\end{aligned}
$$

where $w$ is the bandwidth of each frequency channel and $b_{m n} \in\{0,1\}$ indicates that the $n^{t h}$ user is connected to the $m^{\text {th }}$ FD-UAV or not.

The problem of DL sum-rate maximization, by prescribing a certain minimum requirement for UL transmission rate, can be defined as:

$$
\begin{array}{ll} 
& \max _{P_{m n}^{U A V}, P_{m n}^{U E}, \underline{y_{m}}, b_{m n}} \sum_{m=1}^{M} \sum_{n=1}^{N} R_{m n}^{D L} \\
\text { s.t. } & w \log _{2}\left(1+\frac{P_{m n}^{U E} D_{y m-\alpha_{n}}^{-\alpha_{1}} h_{0} G_{m n}^{U L}}{\sigma_{N}^{2}+\beta P_{m n}^{U A V}+\sigma_{I}^{2}}\right) \geqslant R_{\min }^{U L} \\
& \sum_{n=1}^{N} P_{m n}^{U A V} \leqslant P_{\max }^{U A V}, m \in\{1,2, \ldots, M\} \\
& P_{m n}^{U A V} \geqslant 0 \\
& 0 \leqslant P_{m n}^{U E} \leqslant P_{\max }^{U E} \\
& \sum_{m=1}^{M} b_{m n}=1, n \in\{1,2, \ldots, N\} \\
& \sum_{n=1}^{N} b_{m n} \leqslant T, m \in\{1,2, \ldots, M\},
\end{array}
$$

where $P_{\max }^{U E}$ is the maximum transmitting power of users, $P_{\max }^{U A V}$ is the maximum available power of FD-UAV and $R_{\min }^{U L}$ is minimum UL rate demand. Similar to DL transmission, the problem of UL sum-rate maximization by prescribing a certain minimum requirement for DL transmission rate, can be defined as:

$$
\begin{array}{ll} 
& \max _{P_{m n}^{U A V}, P_{m n}^{U E}, \underline{y_{m}}, b_{m n}} \sum_{m=1}^{M} \sum_{n=1}^{N} R_{m n}^{U L} \\
\text { s.t. } & w \log _{2}\left(1+\frac{P_{m n}^{U A V} D_{y_{m}-z_{n}}^{-\alpha_{0}} h_{0} G_{m n}^{D L}}{\sigma_{N}^{2}+P_{m n}^{U E} D_{x_{n}-z_{n}}^{-\alpha_{0}} h_{0} g+\sigma_{I}^{2}}\right) \geqslant R_{\min }^{D L} \\
& \sum_{n=1}^{N} P_{m n}^{U A V} \leqslant P_{\max }^{U A V}, m \in\{1,2, \ldots, M\} \\
& P_{m n}^{U A V} \geqslant 0 \\
& 0 \leqslant P_{m n}^{U E} \leqslant P_{\max }^{U E} \\
& \sum_{m=1}^{M} b_{m n}=1, n \in\{1,2, \ldots, N\} \\
& \sum_{n=1}^{N} b_{m n} \leqslant T, m \in\{1,2, \ldots, M\}
\end{array}
$$

where $R_{\mathrm{min}}^{D L}$ is the minimum DL rate demand. In addition, the sum of UL and DL sum-rates maximization can be defined as:

$$
\begin{gathered}
\max _{P_{m n}^{U A V}, P_{m E}^{U E}, \underline{y_{m}}, b_{m n}} \sum_{m=1}^{M} \sum_{n=1}^{N} R_{m n}^{D L}+\sum_{m=1}^{M} \sum_{n=1}^{N} R_{m n}^{U L} \\
\text { s.t. } \sum_{n=1}^{N} P_{m n}^{U A V} \leqslant P_{\max }^{U A V}, m \in\{1,2, \ldots, M\} \\
P_{m n}^{U A V} \geqslant 0 \\
0 \leqslant P_{m n}^{U E} \leqslant P_{\max }^{U E} \\
\sum_{m=1}^{M} b_{m n}=1, n \in\{1,2, \ldots, N\} \\
\sum_{n=1}^{N} b_{m n} \leqslant T, m \in\{1,2, \ldots, M\} .
\end{gathered}
$$

In Section 3, at first, we investigate the problem clustering and user association, then, we solve the problem (9), problem (10) and problem (11), respectively. 


\section{Sum-Rate Analysis}

In this section, we aim to maximize DL sum-rate, UL sumrate and sum of DL and UL sum-rates. We divide each problem into two problems. At first, we divide users into $M$ small cells and put a FD-UAV for each cell, then we allocate power to users and FD-UAVs.

\subsection{Clustering and User Association}

We assume that $N$ DL and $N$ UL users are scattered in the considered area. We should put DL users in $M$ clusters and then $b_{m n}$ is determined. It should be noticed that each cluster represents a small cell. Then, we consider the worstcase scenario, i.e., the nearest UL user to the $n^{t h}$ DL user is set as its co-channel user. When users are assigned to the clusters, for each cluster we put a FD-UAV as aerial BS to cover all users which are connected to the $m^{\text {th }}$ FD-UAV.

By using fuzzy c-means clustering (FCM), the membership matrix $U$ and their centers $y$ are generated that determine each user can belong to which cluster and cluster centers, respectively. Each row of matrix $y$ is the location of FD-UAVs and each column of the matrix $U$ is the membership values of each user for clusters. FCM method cannot satisfy our constraint, because there is not any constraint on the number of cluster members and one user can belong to more than one cluster. Hence, we should modify the FCM method to reach our constraint that force small cells to have the same number of users and each user belongs to one cell.

In the first step, we use FCM to determine matrix $U$ and $y$. Then, each user compares the value of $U$ which belongs to itself and maximum value determines that the user belongs to which cluster. Then, we count the number of cluster members, if all clusters have $T$ users, the procedure is done, but if the number of cluster members is not the same, we change the location of cluster centers $(y)$ and again perform the FCM. In order to change cluster centers, we fix the location of cluster centers which have more than $T$ users, and just change cluster centers which gave less than $T$ users as follows:

$$
y_{i}=\delta y_{i}+(1-\delta) y_{j},
$$

where indexes $i$ and $j$ belong to the cluster with the minimum and the maximum number of users, respectively. By these updated locations of cluster centers, again FCM is performed and the number of cluster members is counted until the constraint is satisfied or for a predefined number of iterations. If again constraint is not satisfied by maximum number of iterations, we force users to select clusters. The user with the maximum value of membership, selects its cluster and is removed from the list, then the next user with maximum value selects its cluster. If cluster members are less than $T$, it joins that cluster, but if the cluster reaches $T$ users, the user selects the next cluster. This procedure continues until all users join one cluster. Finally, a FD-UAV is assigned to each cluster and the location of the $m^{\text {th }} \mathrm{FD}$ UAV can be calculated as (13) which is a function of the locations of users and changes with the change of users' locations.

$$
\underline{y_{m}}=\sum_{n=1}^{N} b_{m n} \underline{z_{n}} / \sum_{n=1}^{N} b_{m n} .
$$

This modified FCM method is represented in Algorithm 1.

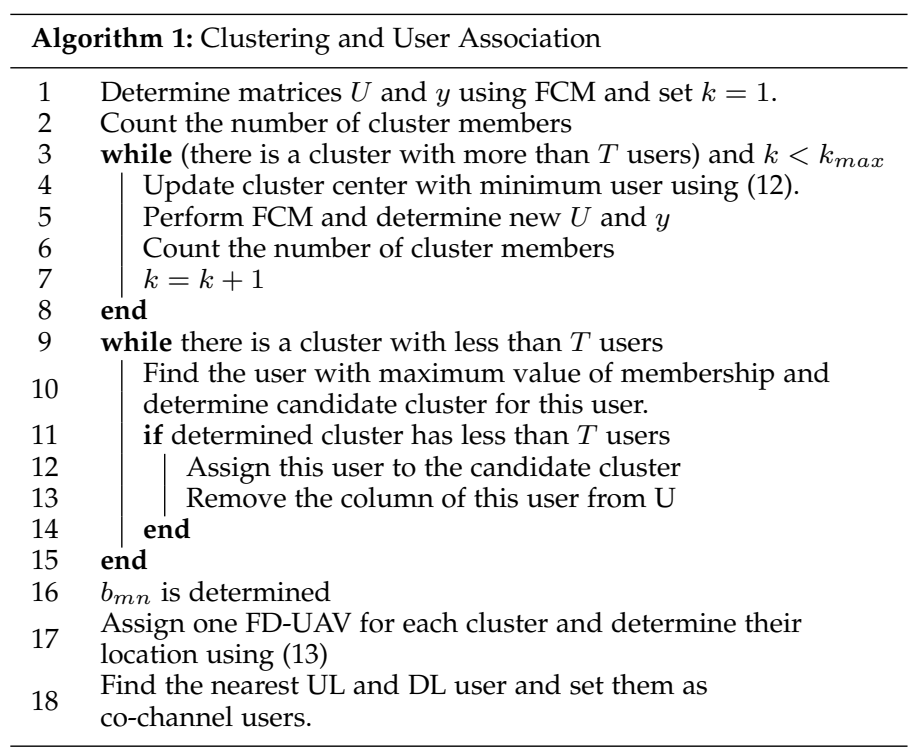

\subsection{Scenario 1: DL Sum-Rate Analysis}

By substituting $b_{m n}$ and $y_{m}$ which are calculated by Algorithm 1 into (9) and some manipulations, problem (9) can be rewritten as (14).

$$
\begin{aligned}
\max _{P_{m n}^{U A V}, P_{m n}^{U E}} & \sum_{m=1}^{M} \sum_{n=1}^{N} R_{m n}^{D L} \\
\text { s.t. } & \frac{\left(2^{\frac{R_{\min }^{U L}}{w}}-1\right)\left(\sigma_{N}^{2}+\beta P_{m n}^{U A V}+\sigma_{I}^{2}\right)}{D_{y_{m}-x_{n}}^{-\alpha_{1}} h_{0} G_{m n}^{U L}} \leqslant P_{m n}^{U E} \leqslant P_{\max }^{U E} \\
& \sum_{n=1}^{N} P_{m n}^{U A V} \leqslant P_{\max }^{U A V}, m \in\{1,2, \ldots, M\} \\
& P_{m n}^{U A V} \geqslant 0
\end{aligned}
$$

In order to maximize the DL sum-rate, it is optimal for all UL users to transmit with the minimum power to satisfy its minimum rate demand, hence, the constraint changes to the equality. In addition, the upper bound of the transmitting power of users is applied to the transmitting power of FDUAV as follows:

$$
\begin{gathered}
\max _{P_{m n}^{U A V}, P_{m n}^{U E}} \sum_{m=1}^{M} \sum_{n=1}^{N} R_{m n}^{D L} \\
\text { s.t. } \quad P_{m n}^{U E}=\frac{\left(2^{\frac{R_{\text {min }}^{U L}}{w}}-1\right)\left(\sigma_{N}^{2}+\beta P_{m n}^{U A V}+\sigma_{I}^{2}\right)}{D_{y_{m}-x_{n}}^{-\alpha} h_{0} G_{m n}^{U L}} \\
\quad \sum_{n=1}^{N} P_{m n}^{U A V} \leqslant P_{\max }^{U A V}, m \in\{1,2, \ldots, M\} \\
0 \leqslant P_{m n}^{U A V} \leqslant \frac{1}{\beta}\left(\frac{P_{\max }^{U E} D_{y_{m}-x_{n}}^{-\alpha_{1}} h_{0} G_{m n}^{U L}}{2^{\frac{R_{\min }^{U L}}{w}}-1}-\sigma_{N}^{2}-\sigma_{I}^{2}\right) .
\end{gathered}
$$

By substituting $P_{m n}^{U E}$ in the optimization problem, the first constraint is satisfied and the optimization problem can 
be rewritten as:

$$
\begin{aligned}
\min _{P_{m n}^{U A V}}- & \sum_{m=1}^{M} \sum_{n=1}^{N} b_{m n} w \log _{2}\left(1+A^{D L}\right) \\
\text { s.t. } \quad & 0 \leqslant P_{m n}^{U A V} \leqslant \frac{1}{\beta}\left(\frac{P_{\max }^{U E} D_{y_{m}-x_{n}}^{-\alpha_{1}} h_{0} G_{m n}^{U L}}{R_{U L}^{*}}-\sigma^{2}\right) \\
& \sum_{n=1}^{N} P_{m n}^{U A V} \leqslant P_{\max }^{U A V}, m \in\{1,2, \ldots, M\},
\end{aligned}
$$

where $A^{D L}=\frac{P_{m n}^{U A V} D_{y_{m}-z_{n}}^{-\alpha_{1}} D_{y_{m}-x_{n}}^{-\alpha_{1}} h_{0} G_{m n}^{D L} G_{m n}^{U L}}{\sigma^{2} D_{y_{m}-x_{n}}^{-\alpha_{1}} G_{m n}^{U L}+R_{U L}^{*}\left(\sigma^{2}+\beta P_{m n}^{U A V}\right) D_{x_{n}-z_{n}}^{-\alpha_{2}} g}$, $R_{U L}^{*}=2^{\frac{R_{\min }^{U L}}{w}}-1$ and $\sigma^{2}=\sigma_{N}^{2}+\sigma_{I}^{2}$.

In (16) the objective function is non-convex, therefore, a closed-form solution is not known for it. In order to solve (16), we rewrite sum-rate as the difference of two concave functions by using properties of logarithmic functions. Therefore, a successive convex algorithm is developed by leveraging the D.C programming [25]. Hence, the problem (16) can be written as a D.C. function, i.e., $f=g-h$. The D.C. programming approximates $f$ by $\widetilde{f}=g-\widetilde{h}$, where $\widetilde{h}$ is the first-order Taylor's series approximation of $h$ [26]. Low complexity, guaranteeing suboptimal solution and obtaining closed-form solutions are some advantages of using D.C. programming.

We consider $P_{m n}^{U A V(0)}$ as an initial value and set $i=0$. In addition, we define an auxiliary function $\widetilde{f}_{1}\left(P_{m n}^{U A V(i)}\right)$ as follows:

$$
\begin{aligned}
& \tilde{f}_{1}\left(P_{m n}^{U A V(i)}\right) \triangleq g_{1}\left(P_{m n}^{U A V}\right)-\widetilde{h_{1}}\left(P_{m n}^{U A V(i)}\right) \\
& =g_{1}\left(P_{m n}^{U A V}\right)-h_{1}\left(P_{m n}^{U A V(i)}\right)-e_{1}\left(P_{m n}^{U A V(i)}\right),
\end{aligned}
$$

where $g_{1}\left(P_{m n}^{U A V}\right), h_{1}\left(P_{m n}^{U A V(i)}\right)$, and $e_{1}\left(P_{m n}^{U A V(i)}\right)$ are defined in 18, 19) and (20, respectively:

$$
\begin{aligned}
& g_{1}\left(P_{m n}^{U A V}\right)=-\sum_{m=1}^{M} \sum_{n=1}^{N} b_{m n} w \\
& \log _{2}\left(P_{m n}^{U A V} D_{y_{m}-z_{n}}^{-\alpha_{1}} D_{y_{m}-x_{n}}^{-\alpha_{1}} h_{0} G_{m n}^{D L} G_{m n}^{U L}\right. \\
& \left.+\sigma^{2} D_{y_{m}-x_{n}}^{-\alpha_{1}} G_{m n}^{U L}+R_{U L}^{*}\left(\sigma^{2}+\beta P_{m n}^{U A V}\right) D_{x_{n}-z_{n}}^{-\alpha_{2}} g\right), \\
& h_{1}\left(P_{m n}^{U A V}{ }^{(i)}\right)=-\sum_{m=1}^{M} \sum_{n=1}^{N} b_{m n} w \\
& \log _{2}\left(\sigma^{2} D_{y_{m}-x_{n}}^{-\alpha_{1}} G_{m n}^{U L}+R_{U L}^{*}\left(\sigma^{2}+\beta P_{m n}^{U A V}{ }^{(i)}\right) D_{x_{n}-z_{n}}^{-\alpha_{2}} g\right),
\end{aligned}
$$

$$
\begin{aligned}
& e_{1}\left(P_{m n}^{U A V}{ }^{(i)}\right)=-\sum_{m=1}^{M} \sum_{n=1}^{N} \frac{b_{m n} w}{\ln 2}
\end{aligned}
$$

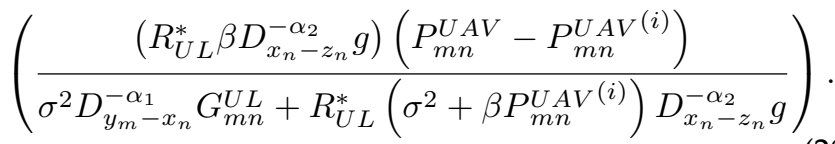

Then, we solve the optimization problem 21.

$$
\begin{array}{ll}
P_{m n}^{U A V}(i+1)= & \arg \min _{P_{m n}^{U A V}} \tilde{f}_{1}\left(P_{m n}^{U A V}{ }^{(i)}\right) \\
\text { s.t. } \quad & 0 \leqslant P_{m n}^{U A V} \leqslant \frac{1}{\beta}\left(\frac{P_{\max }^{U E} D_{y_{m}-x_{n}}^{-\alpha_{1}} h_{0} G_{m n}^{U L}}{R_{U L}^{*}}-\sigma^{2}\right) \\
& \sum_{n=1}^{N} P_{m n}^{U A V} \leqslant P_{\max }^{U A V}, m \in\{1,2, \ldots, M\} .
\end{array}
$$

Then, we set $i=i+1$ and repeat this procedure until the convergence or for a predefined number of iterations. This procedure is represented in Algorithm 2. In this algorithm, the major complexity lies in solving the optimization problem (21). The complexity of solving the problem (21) by using the standard interior point method is $\mathcal{O}\left(I_{i t} M^{3} N^{3}\right)$ where $M, N$ and $I_{i t}$ denote the number of UAVs (or number of small cells), number of users in each cell and the total number of iterations, respectively. As mentioned before, the proposed algorithm is suboptimal, hence, we will show the gap between the proposed algorithm and optimal solution in simulations of Section 4 In addition, the optimality and feasibility of the proposed algorithm are presented in Section 3.5 .

\subsection{Scenario 2: UL Sum-Rate Analysis}

By substituting $b_{m n}$ and $y_{m}$ which are calculated by Algorithm 1 into (10) and some manipulations, problem (10) can be rewritten as (22).

$$
\begin{gathered}
\max _{\substack{P_{m n}^{U A V}, P_{m n}^{U E} \\
\text { s.t. }}} \sum_{m=1}^{M} \sum_{n=1}^{N} R_{m n}^{U L} \\
\quad \sum_{n=1}^{R_{D L}^{*}\left(\sigma^{2}+P_{m n}^{U E} D_{x_{n}-z_{n}}^{-\alpha_{0}} h_{0} g\right)} \\
D_{y_{m}-z_{n}}^{-h_{0} G_{m n}^{D L}} P_{m n}^{U A V} \leqslant P_{\max }^{U A V}, m \in\{1,2, \ldots, M\} \\
0 \leqslant P_{m n}^{U E} \leqslant P_{\max }^{U E},
\end{gathered}
$$

where $R_{D L}^{*}=2^{\frac{R_{\mathrm{min}}^{D L}}{w}}-1$. In order to maximize the UL sumrate, it is optimal for FD-UAV to transmit with the minimum power to satisfy its minimum rate demand, therefore, the optimization problem changes as follows:

$$
\begin{aligned}
& \max _{P_{m n}^{U A V}, P_{m n}^{U E}} \sum_{m=1}^{M} \sum_{n=1}^{N} R_{m n}^{U L} \\
& \text { s.t. } \quad P_{m n}^{U A V}=\frac{R_{D L}^{*}\left(\sigma^{2}+P_{m n}^{U E} D_{x_{n}-z_{n}}^{-\alpha_{2}} h_{0} g\right)}{D_{y_{m}-z_{n}}^{-\alpha_{0}} G_{m n}^{D L}} \\
& \begin{array}{c}
\sum_{n=1}^{N} \frac{R_{D L}^{*}\left(\sigma^{2}+P_{m n}^{U E} D_{x_{n}-z_{n}}^{-\alpha_{2}} h_{0} g\right)}{D_{y_{m}-z_{n}}^{-\alpha_{1}} h_{m} G_{m n}^{D L}} \leqslant P_{\max }^{U A V}, m \in\{1,2, \ldots, M\} \\
0 \leqslant P_{m E}^{U E} \leqslant P_{\max }^{U E}
\end{array}
\end{aligned}
$$

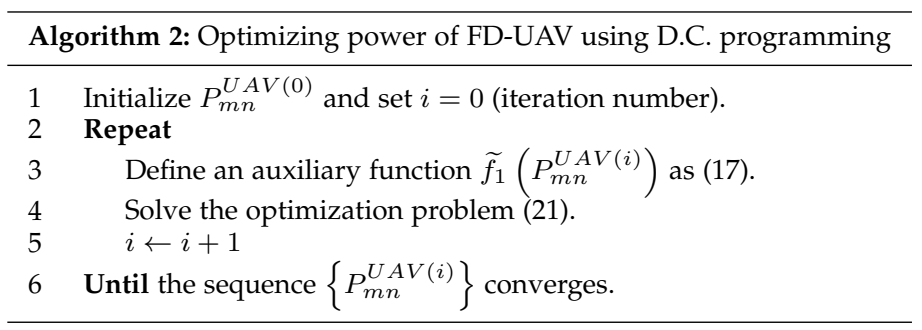


By substituting $P_{m n}^{U A V}$ in the optimization problem [23, the first constraint is satisfied and the optimization problem can be rewritten as:

$$
\begin{gathered}
\min _{P_{m n}^{U E}}-\sum_{m=1}^{M} \sum_{n=1}^{N} b_{m n} w \log _{2}\left(1+A^{U L}\right) \\
\text { s.t. } \quad \sum_{n=1}^{N} \frac{R_{D L}^{*}\left(\sigma^{2}+P_{m n}^{U E} D_{x_{n}-z_{n}}^{-\alpha_{2}} h_{0} g\right)}{D_{y_{m}-z_{n}}^{-\alpha_{0}} G_{m n}^{D L}} \leqslant P_{\max }^{U A V}, m \in\{1,2, \ldots, M\} \\
0 \leqslant P_{m n}^{U E} \leqslant P_{\max }^{U E},
\end{gathered}
$$

where $A^{U L}=\frac{P_{m n}^{U E} D_{y_{m}-x_{n}}^{-\alpha_{1}} D_{y_{m}-z_{n}}^{-\alpha_{1}} h_{0}^{2} G_{m n}^{U L} G_{m n}^{D L}}{\sigma^{2} D_{y_{m}-z_{n}}^{-\alpha_{1}} h_{0} G_{m n}^{D L}+\beta R_{D L}^{*}\left(\sigma^{2}+P_{m n}^{U E} D_{x_{n}-z_{n}}^{-\alpha_{2}} h_{0} g\right)}$. We consider $P_{m n}^{U E(0)}$ as an initial value and set $j=0$. In addition, we define an auxiliary function $\widetilde{f}_{2}\left(P_{m n}^{U E(j)}\right)$ as follows:

$$
\begin{aligned}
& \widetilde{f}_{2}\left(P_{m E^{(j)}}^{U E^{(j)}} \triangleq g_{2}\left(P_{m n}^{U E}\right)-\widetilde{h_{2}}\left(P_{m E^{(j)}}^{U E^{(j)}}\right)\right. \\
& =g_{2}\left(P_{m n}^{U E}\right)-h_{2}\left(P_{m n}^{U E^{(j)}}\right)-e_{2}\left(P_{m n}^{U E^{(j)}}\right),
\end{aligned}
$$

where $g_{2}\left(P_{m n}^{U E}\right), h_{2}\left(P_{m n}^{U E(j)}\right)$ and $e_{2}\left(P_{m n}^{U E(j)}\right)$ are defined in 26, 27) and 28, respectively:

$$
\begin{aligned}
& g_{2}\left(P_{m n}^{U E}\right)=-\sum_{m=1}^{M} \sum_{n=1}^{N} b_{m n} w \\
& \log _{2}\left(P_{m n}^{U E} D_{y_{m}-x_{n}}^{-\alpha_{1}} D_{y_{m}-z_{n}}^{-\alpha_{1}} h_{0}^{2} G_{m n}^{U L} G_{m n}^{D L}\right. \\
& \left.+\sigma^{2} D_{y_{m}-z_{n}}^{-\alpha_{1}} h_{0} G_{m n}^{D L}+\beta R_{D L}^{*}\left(\sigma^{2}+P_{m n}^{U E} D_{x_{n}-z_{n}}^{-\alpha_{2}} h_{0} g\right)\right)
\end{aligned}
$$

$$
\begin{aligned}
& h_{2}\left(P_{m n}^{U E(j)}\right)=-\sum_{m=1}^{M} \sum_{n=1}^{N} b_{m n} w \\
& \log _{2}\left(\sigma^{2} D_{y_{m}-z_{n}}^{-\alpha_{1}} h_{0} G_{m n}^{D L}+\beta R_{D L}^{*}\left(\sigma^{2}+P_{m n}^{U E(j)} D_{x_{n}-z_{n}}^{-\alpha_{2}} h_{0} g\right)\right),
\end{aligned}
$$

$$
\begin{aligned}
& e_{2}\left(P_{m n}^{U E(j)}\right)=-\sum_{m=1}^{M} \sum_{n=1}^{N} \frac{b_{m n} w}{\ln 2} \\
& \left(\frac{\left(\beta R_{D L}^{*} D_{x_{n}-z_{n}}^{-\alpha_{2}} h_{0} g\right)\left(P_{m n}^{U E}-P_{m n}^{U E(j)}\right)}{\sigma^{2} D_{y_{m}-z_{n}}^{-\alpha_{1}} h_{0} G_{m n}^{D L}+\beta R_{D L}^{*}\left(\sigma^{2}+P_{m n}^{U E(j)} D_{x_{n}-z_{n}}^{-\alpha_{2}} h_{0} g\right)}\right) .
\end{aligned}
$$

Then, we solve the optimization problem (29).

$$
\begin{aligned}
& P_{m n}^{U E}{ }^{(j+1)}=\arg \min _{P_{m n}^{U E}} \widetilde{f}_{2}\left(P_{m n}^{U E}(j)\right) \\
& \text { s.t. } \sum_{n=1}^{N} \frac{R_{D L}^{*}\left(\sigma^{2}+P_{m n}^{U E} D_{x_{n}-z_{n}}^{-\alpha_{0}} h_{0}\right)}{D_{y m}^{-\alpha_{1}-z_{n}} h_{0} G_{m n}^{D L}} \leqslant P_{\max }^{U A V}, m \in\{1,2, \ldots, M\} \\
& 0 \leqslant P_{m n}^{U E} \leqslant P_{\max }^{U E} .
\end{aligned}
$$

Then, we set $j=j+1$ and repeat this procedure until the convergence or for a predefined number of iterations. This procedure is the same as Algorithm 2. Similar to the previous part, the major complexity of this algorithm lies in solving the optimization problem (29). The complexity of solving the problem (29) by using the standard interior point method is $\mathcal{O}\left(I_{i t} M^{3} N^{3}\right)$ where $M, N$ and $I_{i t}$ denote the number of UAVs, number of users in each cell and the total number of iterations, respectively.

\subsection{Scenario 3: Sum of UL and DL Sum-Rate Analysis}

By substituting $b_{m n}$ and $y_{m}$ which are calculated by Algorithm 1 in to (11), problem (11) can be rewritten as 30.

$$
\begin{aligned}
& \max _{P_{m n}^{U A V}, P_{m n}^{U E}} \sum_{m=1}^{M} \sum_{n=1}^{N} R_{m n}^{D L}+\sum_{m=1}^{M} \sum_{n=1}^{N} R_{m n}^{U L} \\
& \text { s.t. } \quad \sum_{n=1}^{N} P_{m n}^{U A V} \leqslant P_{\max }^{U A V}, m \in\{1,2, \ldots, M\} \\
& P_{m n}^{U A V} \geqslant 0 \\
& 0 \leqslant P_{m n}^{U E} \leqslant P_{\max }^{U E} .
\end{aligned}
$$

The problem 30 can be written as:

$$
\begin{gathered}
\max _{P_{m n}^{U A V}, P_{m n}^{U E}} \sum_{m=1}^{M} \sum_{n=1}^{N} b_{m n} w\left(\log _{2}\left(1+\frac{s_{1}}{s_{2}}\right)+\log _{2}\left(1+\frac{s_{3}}{s_{4}}\right)\right) \\
\text { s.t. } \sum_{n=1}^{N} P_{m n}^{U A V} \leqslant P_{\max }^{U A V}, m \in\{1,2, \ldots, M\} \\
P_{m n}^{U A V} \geqslant 0 \\
0 \leqslant P_{m n}^{U E} \leqslant P_{\max }^{U E},
\end{gathered}
$$

where $s_{1}=P_{m n}^{U A V} D_{y_{m}-z_{n}}^{-\alpha_{1}} h_{0} G_{m n}^{D L}, s_{2}=\sigma^{2}+$ $P_{m n}^{U E} D_{x_{n}-z_{n}}^{-\alpha_{2}} h_{0} g, s_{3}=P_{m n}^{U E} D_{y_{m}-x_{n}}^{-\alpha_{1}} h_{0} G_{m n}^{U L}$ and $s_{4}=\sigma^{2}+$ $\beta P_{m n}^{U A V}$. By using properties of logarithmic functions, problem (31) can be rewritten as:

$$
\begin{gathered}
\min _{P_{m n}^{U A V}, P_{m n}^{U E}}-\sum_{m=1}^{M} \sum_{n=1}^{N} b_{m n} w \log _{2}\left(\frac{\left(s_{1}+s_{2}\right)\left(s_{3}+s_{4}\right)}{s_{2} s_{4}}\right) \\
\text { s.t. } \quad \sum_{n=1}^{N} P_{m n}^{U A V} \leqslant P_{\max }^{U A V}, m \in\{1,2, \ldots, M\} \\
P_{m A V}^{U A V} \geqslant 0 \\
0 \leqslant P_{m n}^{U E} \leqslant P_{\max }^{U E} .
\end{gathered}
$$

For jointly optimizing the transmitting power of users and transmitting power of FD-UAV using D.C. programming, we consider $P_{m n}^{U A V(0)}$ and $P_{m n}^{U E(0)}$ as initial values and set $i=0$. In addition, we define an auxiliary function $\widetilde{f}_{3}\left(P_{m n}^{U A V(i)}, P_{m n}^{U E(i)}\right)$ as follows:

$\widetilde{f}_{3}\left(P_{m n}^{U A V(i)}, P_{m n}^{U E(i)}\right)=$

$-\sum_{m=1}^{M} \sum_{n=1}^{N} b_{m n} w \log _{2}\left(\left(s_{1}+s_{2}\right)\left(s_{3}+s_{4}\right)\right)$

$+\sum_{m=1}^{M} \sum_{n=1}^{N} b_{m n} w \log _{2}\left(s_{5}\right)+\sum_{m=1}^{M} \sum_{n=1}^{N} b_{m n} w$

$\left(\frac{P_{m n}^{U A V}-P_{m n}^{U A V(i)}}{\ln 2} \times \frac{\sigma^{2} \beta+\beta P_{m n}^{U E(i)} D_{x_{n}-z_{n}}^{-\alpha_{2}} h_{0} g}{s_{5}}\right)$

$+\sum_{m=1}^{M} \sum_{n=1}^{N} b_{m n} w$

$\left(\frac{P_{m n}^{U E}-P_{m n}^{U E(i)}}{\ln 2} \times \frac{\sigma^{2} D_{x_{n}-z_{n}}^{-\alpha_{2}} h_{0} g+\beta P_{m n}^{U A V(i)} D_{x_{n}-z_{n}}^{-\alpha_{2}} h_{0} g}{s_{5}}\right)$,

where $s_{5}=\sigma^{4}+\sigma^{2} \beta P_{m n}^{U A V(i)}+\sigma^{2} P_{m n}^{U E(i)} D_{x_{n}-z_{n}}^{-\alpha_{2}} h_{0} g+$ $\beta P_{m n}^{U A V(i)} P_{m n}^{U E(i)} D_{x_{n}-z_{n}}^{-\alpha_{2}} h_{0} g$. Then, we solve the optimiza- 
tion problem 34 .

$$
\begin{gathered}
{\left[P_{m n}^{U A V}{ }^{(i+1)}, P_{m n}^{U E}{ }^{(i+1)}\right]=} \\
\arg \min _{P_{m n}^{U A}, P_{m n}^{U E}} \widetilde{f}_{3}\left(P_{m n}^{U A V(i)}, P_{m n}^{U E(i)}\right) \\
\text { s.t. } \sum_{n=1}^{N} P_{m n}^{U A V} \leqslant P_{\max }^{U A V}, m \in\{1,2, \ldots, M\} \\
P_{m n}^{U A V} \geqslant 0 \\
0 \leqslant P_{m n}^{U E} \leqslant P_{\max }^{U E} .
\end{gathered}
$$

Then, we set $i=i+1$ and repeat this procedure until the convergence or for a predefined number of iterations. This procedure is the same as Algorithm 2. In this algorithm, the major complexity lies in solving the optimization problem (34). The complexity of solving the problem (34) by using the standard interior point method is $\mathcal{O}\left(I_{i t}(2 M)^{3} N^{3}\right)$ where $M, N$ and $I_{i t}$ denote the number of UAVs, number of users in each cell and the total number of iterations.

\subsection{Optimality and Feasibility}

We should mention that the proposed algorithm obtains a locally optimal solution. From Proposition 3 in [27], in order to show the optimality of the proposed algorithm, Lemma 1 can be written as follows:

Lemma 1: Let $\mathcal{F}$ be a maximization problem with differentiable objective function $f_{0}(x)$ and constraints $f_{i}(x) \geqslant 0, i=$ $0,1, \ldots, I$ with a compact feasible set. Let $\mathcal{G} \mid$ be a maximization problem with differentiable objective $g_{0, j}(x)$ and constraints $g_{i, j}(x) \geqslant 0, i=0,1, \ldots, I$ with a compact feasible set and optimal solution $x_{j}^{*}$. Assume that for all values of $i$ and $j, g_{i, j}($.$) satisfies$ the following properties:

- $g_{i, j}(x) \leqslant f_{i}(x) \forall x$

- $g_{i, j}\left(x_{j-1}^{*}\right)=f_{i}\left(x_{j-1}^{*}\right)$.

The sequence $\left\{f_{0}\left(x_{j}^{*}\right)\right\}$ is monotonically increasing and converges to a finite limit $g$. Now, assume that the following property is also satisfied:

- $\nabla g_{i, j}\left(x_{j-1}^{*}\right)=\nabla f_{i}\left(x_{j-1}^{*}\right)$.

Then, under suitable constraints qualifications, every limit point of $\{x\}_{j}$ that achieves the objective value $g$, fulfills the KarushKuhn-Tucker (KKT) conditions of the original problem $\mathcal{F}$ [27].

According to Lemma 1, by considering the first-order Taylor's series as a sequence of approximate problem, one can generate a sequence of feasible points $x_{j}$ that monotonically increases the value of the original objective $f_{0}$ and converges to a locally optimal solution.

To ensure the feasibility of the problem (14), we employ the feasibility checking problem [28 by minimizing the transmit power of FD-UAV subject to constraints 14b and (14d). If the minimal sum power of FD-UAV is larger than $P_{\max }^{U A V}$, problem (14) is infeasible. Considering that $P_{m n}^{U A V *}$ is the minimal value of $P_{m n}^{U A V}$ according to $114 \mathrm{~b}$, the feasibility checking problem is equivalent to obtain the minimum value $v^{*}$ of $\sum_{n=1}^{N} P_{m n}^{U A V}$ assuming $P_{m n}^{U E}=0, H_{0}=H_{L}$, and constraints are satisfied. The sum power minimization problem can be solved by an exhaustive search algorithm. The optimal transmit power of users can be obtained via the interior point method. Consequently, problem (14) is feasible if and only if $P_{\max }^{U A V} \geqslant v^{*}$.

\section{Simulation Results}

In this section, the presented system and resource allocation algorithms in FD-UAV enabled small cell networks will be validated by computational simulation. We consider a square area with the dimension of $300 \times 300\left(\mathrm{~m}^{2}\right)$ in which, there is a network consisting $N=45 \mathrm{UL}$ users and $N=45$ DL users which are uniformly distributed in the square area. In the first step, we perform Algorithm 1 and put users in $M=3$ small cells, then, we investigate the problem of resource allocation for the scenario of 3.2 (labeled 'SCN1'), the scenario of 3.3 (labeled 'SCN2') and the scenario of 3.4 (labeled 'SCN3'). In this section, the total sum-rate means the sum of UL and DL sum-rates. We compare the results of our proposed scenario (labeled as 'FDUAV') with the following scenarios: UAV with HD transmission used in [21] (labeled as 'HDUAV'), ground BS with FD transmission used in [19] (labeled as 'FDGBS') and ground BS with HD transmission (labeled as 'HDGBS'). The simulation results are averaged for 1000 different random realizations. We assume that the FD-UAV altitude is bounded between $50 \mathrm{~m}$ and $500 \mathrm{~m}$. We set $\Theta_{0}=\pi / 4, \alpha_{1}=2, \alpha_{2}=4$, $\sigma_{N}^{2}=-50 \mathrm{~dB}, \sigma_{I}^{2}=0.1 \sigma_{N}^{2}, \beta=-95 \mathrm{~dB}, R_{\min }^{U L}=1 \mathrm{kbits} / \mathrm{s}$, $R_{\min }^{D L}=1 \mathrm{Mbits} / \mathrm{s}, P_{\max }^{U A V}=42 \mathrm{dBm}, P_{\max }^{U E}=24 \mathrm{dBm}$, $G_{0}=2.2846, h_{0}=1.42 \times 10^{-4}$ and the total bandwidth of each cell is $15 \mathrm{MHz}$,. In addition, we assume that the height of ground BS is $30 \mathrm{~m}$ and the path-loss exponent between ground BS and users is more than $\alpha_{1}$ and less than $\alpha_{2}$.

The effect of minimum rate demand for FD-UAV is investigated in Fig. 2 At first, we assume that the minimum rate demand for UL and DL users is $1 \mathrm{kbits} / \mathrm{s}$ (Fig. 2a. In this case, the total sum-rate of SCN 3 is about

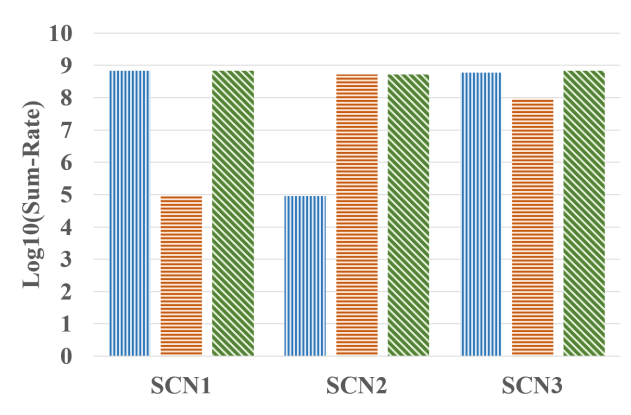

III DL Sum-Rate $\equiv$ UL Sum-Rate s Sum of UL and DL Sum-Rate

(a) Sum-Rate Comparison when $R_{\min }^{U L}=R_{\min }^{D L}=1 \mathrm{kbits} / \mathrm{s}$

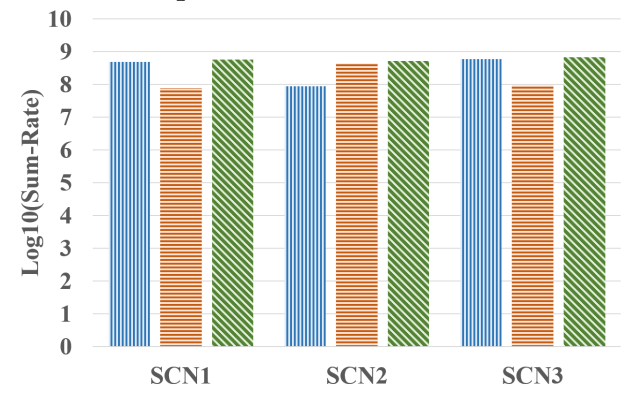

III DL Sum-Rate $\equiv$ UL Sum-Rate $\mathrm{s}$ Sum of UL and DL Sum-Rate

(b) Sum-Rate Comparison when $R_{\min }^{U L}=R_{\min }^{D L}=1 \mathrm{Mbits} / \mathrm{s}$

Fig. 2: Effect of minimum rate demand on sum-rate for FDUAV. 
$688 \mathrm{Mbits} / \mathrm{s}$, while the total sum-rate of SCN 1 and SCN 2 are $687 \mathrm{Mbits} / \mathrm{s}$ and $528 \mathrm{Mbits} / \mathrm{s}$, respectively. In SCN 1 , the DL sum-rate is about $10^{3}$ times more than the UL sum-rate, while in SCN 2, the UL sum-rate is about $10^{3}$ times more than the DL sum-rate, and in SCN 3, both UL and DL sum-rates are almost the same. Now, we assume that the minimum rate demand for UL and DL users is $1 \mathrm{Mbits} / \mathrm{s}$ (Fig. 2b). In this case, the total sum-rates of all three scenarios is almost the same (about $0.6 \mathrm{Gbits} / \mathrm{s}$ ). In addition in all three scenarios of Fig. 2b, the difference between UL and DL sum-rates is very low in comparison with Fig. $2 \mathrm{a}$, because we increase the minimum rate demand and the amount of UL sum-rate and DL sum-rate become closer together.

In Fig. 3 we compare the DL sum-rate of aerial and ground BSs as a function of UL minimum rate demand. As expected, HD-UAV and HD-GBS are not affected by increasing minimum rate demand, while DL sum-rate of FD-UAV and FD-GBS decrease. DL sum-rate of FD-UAV is more than HD-UAV, if minimum rate demand is less than $2 M$ bits $/ s$, if minimum rate demand increases to more than $2 M b i t s / s$, HD mode outperforms FD ones in UAVs. Moreover, Fig. 3 indicates that the DL sum-rate of FD-GBS is highly affected by UL users and in this case, HD mode performs better than FD mode.

In Fig. 4 we compare UL sum-rate of aerial and ground BSs as a function of DL minimum rate demand. As expected,

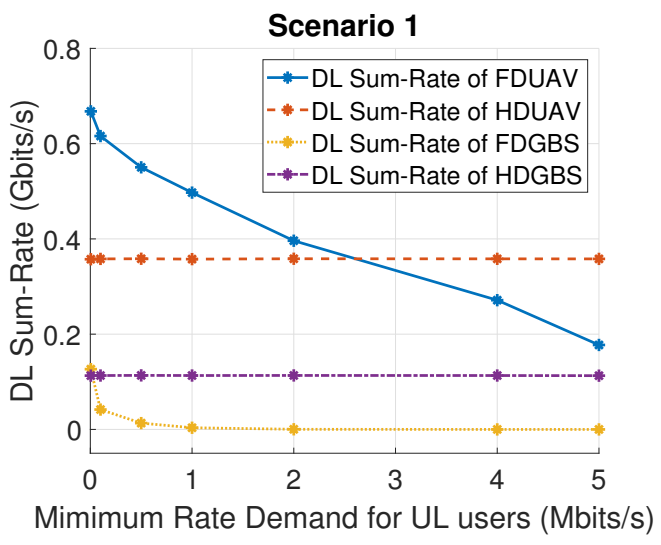

Fig. 3: DL sum-rate against rate in SCN 1.

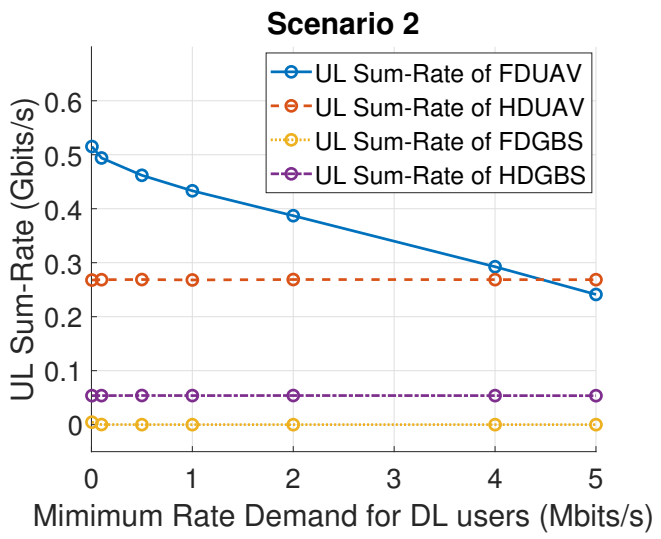

Fig. 4: UL sum-rate for different rates in SCN 2.
HD-UAV and HD-GBS are not affected by increasing minimum rate demand, while UL sum-rate of FD-UAV and FDGBS are decreased. Fig. 4 indicates that the UL sum-rate of FD-GBS is highly affected by DL users and in this case, HD mode performs better than FD mode. In addition, DL sumrate of FD-UAV is more than HD-UAV, if the minimum rate demand is less than $4 M b i t s / s$, if minimum rate demand increases more than $4 \mathrm{Mbits} / \mathrm{s}$, HD mode outperforms FD ones in UAVs.

In Fig. 5 we show the outage probability of UL and DL users in SCN 3. It can be seen that UL users are more sensitive to rate threshold in comparison with DL users. Moreover, FD-UAV outperforms FD-GBS and can prepare a better experience for users. Moreover, by increasing the rate threshold more than $10 \mathrm{Mbits} / \mathrm{s}$, the outage probability tends to one for all users.

Due to the importance of the SIC factor in FD systems, in Fig. 6, Fig. 7 and Fig. 8 we show how SIC factor affects the sum-rate. Fig. 6 indicates that aerial BS outperforms ground BS in terms of DL sum-rate. In addition, it can be seen that when the SIC factor is less than $-50 d B$, FD-UAV outperforms HD-UAV, and after this point, it is better to use HD mode, while for ground BSs, SIC factor should be less than $-90 d B$ in order to use FD mode. Therefore, FD-UAVs can achieve more DL sum-rate with less SIC factor and less complexity.

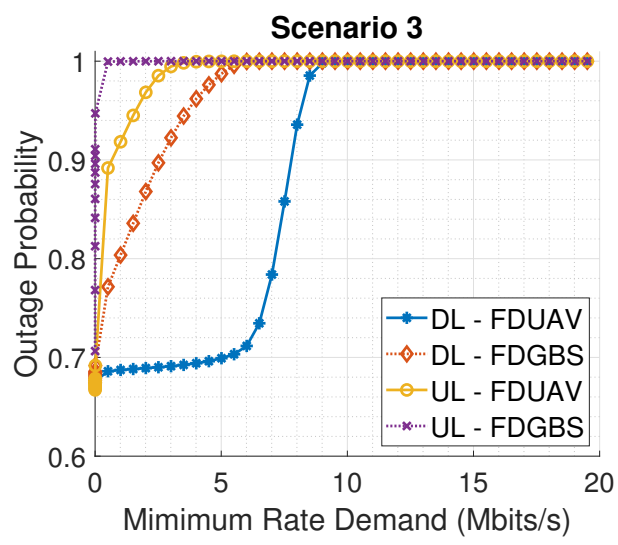

Fig. 5: Outage probability for different rate thresholds in SCN 3.

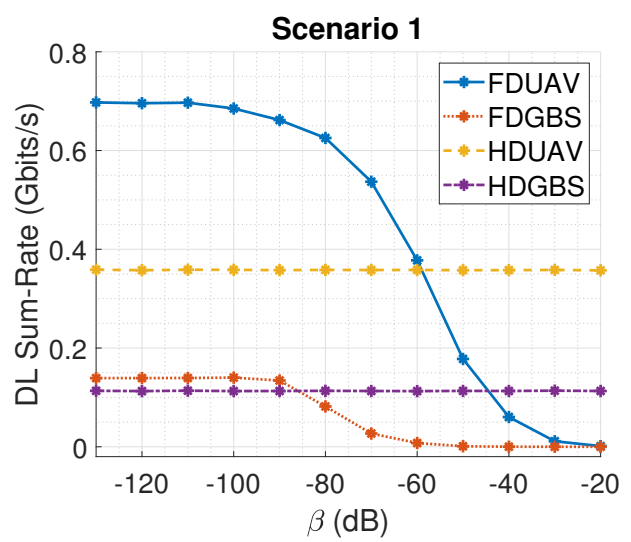

Fig. 6: DL sum-rate for different SIC factors in SCN 1. 
Fig. 7 7 shows that the performance of FD-GBS is not comparable with FD-UAV and in the UL sum-rate maximization scenario (SCN2), FD-GBS is highly affected by interference signals, because of minimum DL rate demand. In addition, it can be seen that FD-UAV outperforms HD-UAV if the SIC factor is less than $-80 d B$, otherwise, HD-UAV achieves more UL sum-rate. Moreover, when SIC factor is higher than $-60 d B$, both HD-UAV and HD-GBS outperform FD-UAV.

As can be seen in Fig. 8 in scenarios which it is needed to maximize the total sum-rate, SIC factor should be less than $-100 d B$ for a reasonable employing of FD-UAV, otherwise, HD-UAV outperforms FD-UAV. Moreover, unlike SCN1 and SCN2, FD-UAV in SCN3 needs a better SIC factor to outperform HD-UAV. In addition, Fig. 8 indicates that the SIC factor does not affect the total sum-rate considerably.

In order to show the gap between the proposed and optimal solution, we compare our proposed iterative method of Scenario 1 with the exhaustive search method in Table 3 It can be seen that the sum-rate of exhaustive search method is slightly higher than that of the proposed method, which indicates that the proposed method approaches the globally optimal solution and the gap between the proposed and optimal solution is very small.

In Fig. 9 we investigate the effect of the maximum power of each BS on the DL sum-rate in SCN1. By increasing the maximum power, DL sum-rate increases; at first, the increment is fast. After that, the increment is slow and the

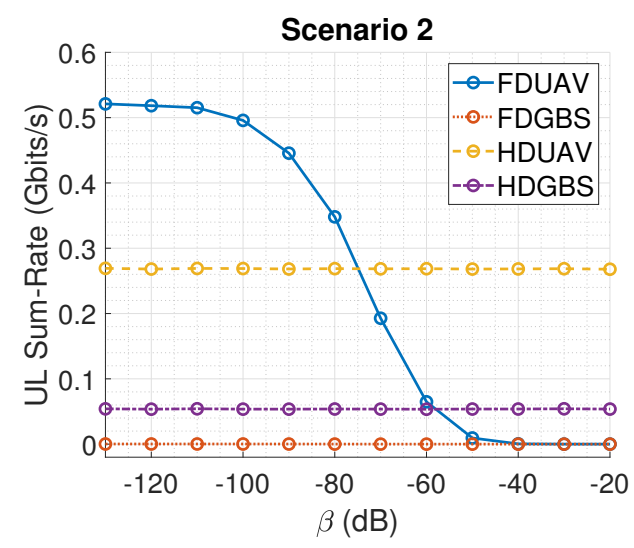

Fig. 7: UL sum-rate for different SIC factors in SCN 2.

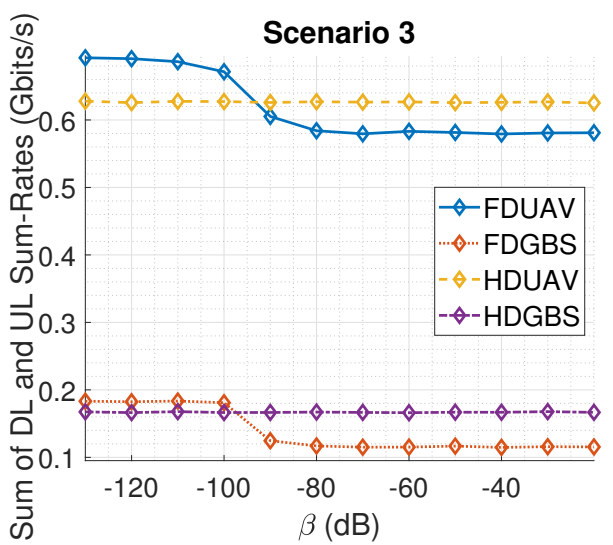

Fig. 8: Total sum-rate for different SIC factors in SCN 3.
TABLE 3: Comparison of DL sum-rate (in Mbits/s) in the proposed method of Scenario 1 and exhaustive search.

\begin{tabular}{|c|c|c|}
\hline$\beta$ & FD-UAV (Proposed) & Exhaustive Search \\
\hline$-90 d B$ & 661.5027 & 661.7064 \\
\hline$-80 d B$ & 625.4408 & 625.5210 \\
\hline$-70 d B$ & 536.9137 & 536.9957 \\
\hline$-60 d B$ & 377.5836 & 377.6126 \\
\hline$-50 d B$ & 177.8941 & 177.9344 \\
\hline$-40 d B$ & 60.2434 & 60.2704 \\
\hline
\end{tabular}

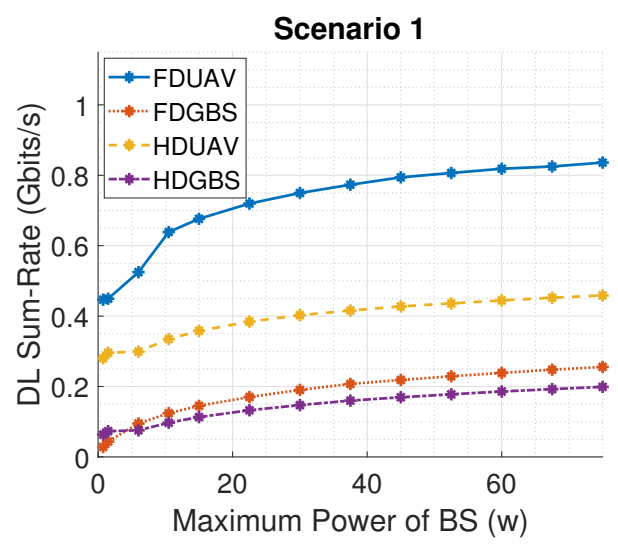

Fig. 9: Effect of maximum power of each BS on the sum-rate in SCN1.

amount of sum-rate is almost fixed. In almost all cases, FD mode outperforms HD mode because of the appropriate SIC factor.

The effect of the maximum power of each BS on the total sum-rate in SCN3 is presented in Fig. 10 It can be seen that by increasing the maximum power of FD BSs, the total sum-rate increases rapidly until about $15 \mathrm{w}$ and after this point, the total sum-rate is almost fixed and does not change considerably. In addition, Fig. 10 shows that in SCN3, just for special values of power, FD-UAV outperforms HD-UAV and in almost all situations, HD mode outperforms FD mode because of interference signals and SIC factor. As mentioned before, we can see from Fig. 8 that if the SIC factor can be less than $-100 d B$, the total sum-rate of FD mode becomes more than HD mode, otherwise, HD mode outperforms FD one in term of total sum-rate.

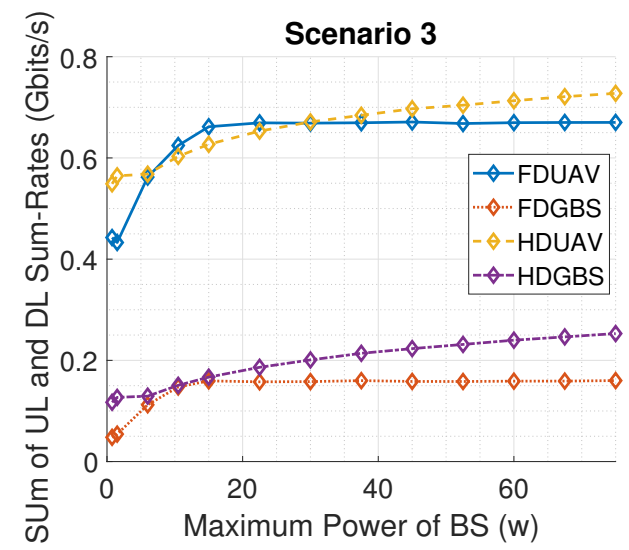

Fig. 10: Effect of maximum power of each BS on the sumrate in $\mathrm{SCN} 3$. 
Fig. 11 presents the effect of half-power beamwidth on the total sum-rate. It can be seen that by increasing $\Theta$, the total sum-rate increases until $\Theta=15^{\circ}$, because by increasing half-power beamwidth, more users are covered by FD$\mathrm{UAVs}$ and total sum-rate increases. By increasing $\Theta$ more than $15^{\circ}$, the total sum-rate does not change considerably until $\Theta=65^{\circ}$, at this point, the total sum-rate reaches its maximum value. By increasing $\Theta$ more than $65^{\circ}$, total sumrate decreases, because FD-UAVs are in the coverage area of each other and receive strong co-channel interferences from each other. Moreover, SCN3 and SCN1 achieve more total sum-rate in comparison with SCN2.

In the last simulation, we assume that $M$ is the number of small cells and there are $15 \mathrm{M}$ UL users and $15 \mathrm{M}$ DL users scattered in a square area of $100 M \times 100 M\left(m^{2}\right)$. In addition, we assume that for each cell $w=15 \mathrm{MHz}$. For a various numbers of cells, we calculate the total sum-rate of the whole network and divide it to the number of cells in order to calculate the average of total sum-rate per cell, and the result is presented in Fig. 12. This figure shows the effectiveness of FD-UAVs in comparison with FD-GBSs. It can be seen that by increasing the number of cells in the adjacency of each other, FD-UAV in SCN3 achieves more average of total sum-rate per cell until $M=3$, while by increasing the number of cells more than 3 cells, FD-UAV in SCN1 achieves more average of total sum-rate per cell and in all cases, FD-UAV in SCN2 achieves less sum-rate in comparison with SCN1 and SCN3.

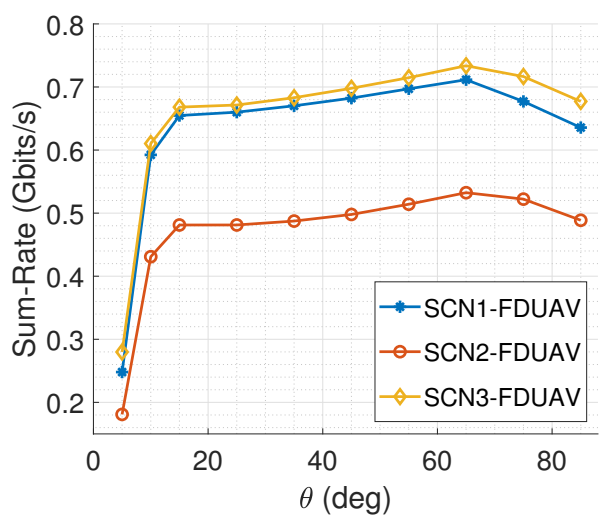

Fig. 11: Effect of half-power beamwidth on total sum-rate.

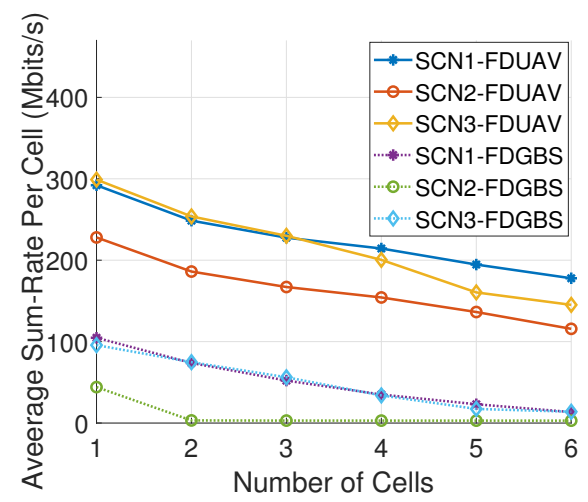

Fig. 12: Average sum-rate for different numbers of cells.

\section{Conclusion}

In this paper, we have investigated the problem of resource allocation for FD-UAVs as aerial BSs and HD cellular users in multi-small cell networks. We assumed that FD-UAVs serve both UL and DL users simultaneously with imperfect SIC. Considering intra-cell and inter-cell interferences, we have formulated the optimization problem of resource allocation for three different scenarios. The optimization problems are non-convex, therefore, we have developed successive convex approximation algorithms by leveraging D.C. programming to find sub-optimal solutions with fairly acceptable computational complexity. Finally, through extensive simulations under various system parameters, and analysis of the optimality, feasibility and complexity of our proposed system, we have shown effectiveness of our proposed system. Simulation Results reveal that SI and SIC factor are the dominant performance limiting factors. In addition, simulation results showed that to employ FDUAVs or HD-UAVs, several factors such as SIC capability, minimum rate demand, density of small cells and power control should be considered. Besides, results reveal how FD transmission enhances the network performance in comparison with HD one. To improve system performance, other issues could be considered such as user pairing, bandwidth optimization and mode selection between HD and FD modes which are left for future works.

\section{REFERENCES}

[1] S. Hayat, E. Yanmaz, and R. Muzaffar, "Survey on unmanned aerial vehicle networks for civil applications: A communications viewpoint," IEEE Communications Surveys \& Tutorials, vol. 18, no. 4, pp. 2624-2661, 2016.

[2] M. Hua, Y. Wang, C. Li, Y. Huang, and L. Yang, "UAV-aided mobile edge computing systems with one by one access scheme," IEEE Trans. Green Communications and Networking, vol. 3, no. 3, pp. 664-678, Sep. 2019.

[3] Z. Yang, W. Xu, and M. Shikh-Bahaei, "Energy efficient UAV communication with energy harvesting," IEEE Transactions on Vehicular Technology, vol. 69, no. 2, pp. 1913-1927, 2020.

[4] A. Fotouhi, H. Qiang, M. Ding, M. Hassan, L. G. Giordano, A. Garcia-Rodriguez, and J. Yuan, "Survey on UAV cellular communications: Practical aspects, standardization advancements, regulation, and security challenges," IEEE Communications Surveys E Tutorials, vol. 21, no. 4, pp. 3417-3442, 2019.

[5] J. Li and Y. Han, "Optimal resource allocation for packet delay minimization in multi-layer UAV networks," IEEE Communications Letters, vol. 21, no. 3, pp. 580-583, 2016.

[6] M. Mozaffari, W. Saad, M. Bennis, Y.-H. Nam, and M. Debbah, "A tutorial on UAVs for wireless networks: Applications, challenges, and open problems," IEEE Communications Surveys \& Tutorials, vol. 21, no. 3, pp. 2334-2360, 2019.

[7] Y. Wang, M. Hua, Z. Liu, D. Zhang, B. Ji, and H. Dai, “UAV-based mobile wireless power transfer systems with joint optimization of user scheduling and trajectory," Mobile Networks and Applications, pp. 1-15, 2019.

[8] Y. Zeng, J. Xu, and R. Zhang, "Energy minimization for wireless communication with rotary-wing UAV," IEEE Transactions on Wireless Communications, vol. 18, no. 4, pp. 2329-2345, 2019.

[9] B. Li, Z. Fei, and Y. Zhang, "UAV communications for 5G and beyond: Recent advances and future trends," IEEE Internet of Things Journal, vol. 6, no. 2, pp. 2241-2263, 2018.

[10] Z. Yuan, J. Jin, L. Sun, K.-W. Chin, and G.-M. Muntean, "Ultrareliable IoT communications with UAVs: A swarm use case," IEEE Communications Magazine, vol. 56, no. 12, pp. 90-96, 2018.

[11] L. Chen, C. Zhong, H. Lin, and Z. Zhang, "Joint user pairing and power allocation design for heavy loaded full-duplex small cell systems," IEEE Transactions on Vehicular Technology, vol. 67, no. 9, pp. 8989-8993, 2018. 
[12] M. Naslcheraghi, S. A. Ghorashi, and M. Shikh-Bahaei, "Fullduplex device-to-device collaboration for low-latency wireless video distribution," in 24th IEEE International Conference on Telecommunications (ICT), 2017, pp. 1-5.

[13] B. Mousavinasab, A. H. Gazestani, S. A. Ghorashi, and M. ShikhBahaei, "Throughput improvement by mode selection in hybrid duplex wireless networks," Wireless Networks, vol. 26, no. 5, pp. 3687-3699, 2020.

[14] T. Riihonen, S. Werner, and R. Wichman, "Mitigation of loopback self-interference in full-duplex MIMO relays," IEEE Transactions on Signal Processing, vol. 59, no. 12, pp. 5983-5993, 2011.

[15] S. Goyal, P. Liu, S. S. Panwar, R. A. Difazio, R. Yang, E. Bala et al., "Full duplex cellular systems: will doubling interference prevent doubling capacity?" IEEE Communications Magazine, vol. 53, no. 5, pp. 121-127, 2015.

[16] Z. Zhang, K. Long, A. V. Vasilakos, and L. Hanzo, "Full-duplex wireless communications: Challenges, solutions, and future research directions," Proceedings of the IEEE, vol. 104, no. 7, pp. 13691409, 2016.

[17] G. Liu, F. R. Yu, H. Ji, V. C. Leung, and X. Li, “In-band fullduplex relaying: A survey, research issues and challenges," IEEE Communications Surveys \& Tutorials, vol. 17, no. 2, pp. 500-524, 2015.

[18] A. Hajihoseini Gazestani, S. Ghorashi, B. Mousavinasab, and M. Shikh-Bahaei, "A survey on implementation and applications of full duplex wireless communications," Physical Communication, vol. 34, pp. $121-134,2019$.

[19] H. Malik, M. Ghoraishi, and R. Tafazolli, "Suboptimal radio resource management for full-duplex enabled small cells," in IEEE International Conference on Communications Workshops (ICC Workshops), 2017, pp. 942-947.

[20] L. Deng, G. Wu, J. Fu, Y. Zhang, and Y. Yang, "Joint resource allocation and trajectory control for uav-enabled vehicular communications," IEEE Access, vol. 7, pp. 132 806-132 815, 2019.

[21] Z. Yang, C. Pan, M. Shikh-Bahaei, W. Xu, M. Chen, M. Elkashlan, and A. Nallanathan, "Joint altitude, beamwidth, location and bandwidth optimization for UAV-enabled communications," IEEE Communications Letters, vol. 22, no. 8, pp. 1716-1719, 2018.

[22] J. Plachy, Z. Becvar, P. Mach, R. Marik, and M. Vondra, "Joint positioning of flying base stations and association of users: Evolutionary-based approach," IEEE Access, vol. 7, pp. 11454$11463,2019$.

[23] L. Zhang, Q. Fan, and N. Ansari, "3-d drone-base-station placement with in-band full-duplex communications," IEEE Communications Letters, vol. 22, no. 9, pp. 1902-1905, 2018.

[24] C. Balanis, Antenna Theory: Analysis and Design, 4th ed. Wiley, 2015.

[25] H. Al-Shatri and T. Weber, "Achieving the maximum sum rate using DC programming in cellular networks," IEEE Transactions on signal processing, vol. 60, no. 3, pp. 1331-1341, 2012.

[26] S. Huberman and T. Le-Ngoc, "Full-duplex MIMO precoding for sum-rate maximization with sequential convex programming," IEEE Transactions on Vehicular Technology, vol. 64, no. 11, pp. 5103$5112,2014$.

[27] A. Zappone, E. Björnson, L. Sanguinetti, and E. Jorswieck, "Globally optimal energy-efficient power control and receiver design in wireless networks," IEEE Transactions on Signal Processing, vol. 65, no. 11, pp. 2844-2859, 2017.

[28] W. Huang, Z. Yang, C. Pan, L. Pei, M. Chen, M. Shikh-Bahaei, M. Elkashlan, and A. Nallanathan, "Joint power, altitude, location and bandwidth optimization for UAV with underlaid D2D communications," IEEE Wireless Communications Letters, vol. 8, no. 2, pp. 524-527, 2018.

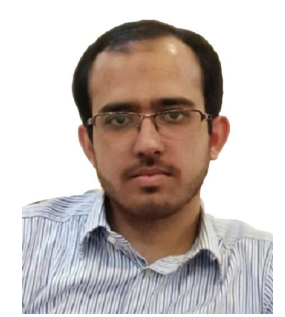

Amirhosein Hajihoseini Gazestani received the B.S. degree in Electrical Engineering at Shahed University, Tehran, Iran, in 2014 and the M.Sc. degree in Telecommunications Engineering from the Shahid Beheshti University (SBU), in 2016. Currently, he is pursuing his $\mathrm{PhD}$. degree in Telecommunications Engineering at SBU, Tehran, Iran. His main research interests include Full-Duplex wireless communications, spectrum sensing, cognitive radio and target localization.

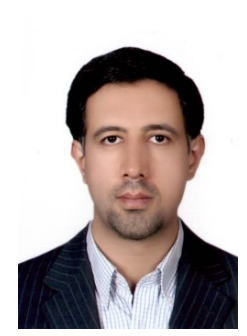

Seyed Ali Ghorashi received his BSc and MSc degrees in Electrical Engineering from the University of Tehran, Iran and his PhD degree from Kings College London, UK. He has worked for Samsung Electronics (UK) Ltd, Shahid Beheshti University and University of East London. $\mathrm{He}$ is a senior member of IEEE, holds three US patents, and has published over 100 technical papers mainly related to the applications of optimization, artificial intelligence and machine learning in localization, internet of things and wireless

communications.

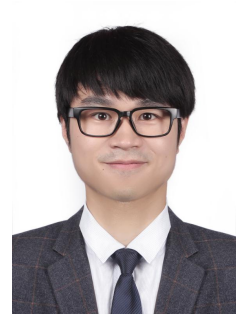

Zhaohui Yang received the B.S. degree in information science and engineering from ChienShiung Wu Honors College, Southeast University, Nanjing, China, in June 2014, the Ph.D. degree in communication and information system with the National Mobile Communications Research Laboratory, Southeast University, Nanjing, China, in May 2018. He is currently a PostDoctoral Research Associate with the Center for Telecommunications Research, Department of Engineering, King's College London, U.K., where he has been involved in the EPSRC SENSE Project. His research interests include UAV, MEC, machine learning, URLLC, energy harvesting, and NOMA. He was a TPC member of IEEE ICC during 2015-2019 and Globecom during 2017-2019.

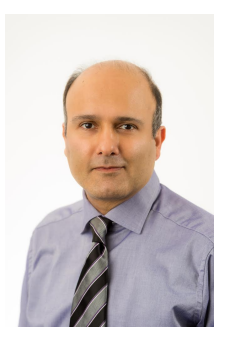

Mohammad Shikh-Bahaei (S'96-M'00-SM'08) received the B.Sc. degree from the University of Tehran, Tehran, Iran, in 1992, the M.Sc. degree from the Sharif University of Technology, Tehran, in 1994, and the Ph.D. degree from the Kings College London, U.K., in 2000. He has worked for two start-up companies, and for National Semiconductor Corporation, Santa Clara, CA, USA (now part of Texas Instruments Inc.), on the design of third-generation mobile handsets, for which he has received three U.S. patents as inventor and co-inventor, respectively. In 2002, he joined the Kings College London as a Lecturer, where he is currently a full Professor. $\mathrm{He}$ has since authored or co-authored numerous journal and conference articles. He has been involved in research in the area of wireless communications and signal processing for 25 years both in academic and industrial organizations. His research interests include resource allocation in full-duplex and cognitive dense networks, visual data communications over the loT, applications in healthcare, and communication protocols for autonomous vehicle/drone networks. He is a fellow of the IET and the Founder and the Chair of the Wireless Advanced (formerly SPWC) Annual International Conference from 2003 to 2018. 